\title{
CYP2E1 changes the biological function of gastric cancer cells via the PI3K/Akt/mTOR signaling pathway
}

\author{
RUI-YING WANG, XIAO-WEI CHEN, WEN-WEN ZHANG, \\ FEI JIANG, MENG-QI LIU and XIAO-BING SHEN
}

\begin{abstract}
Key Laboratory of Environmental Medicine Engineering, Ministry of Education, School of Public Health, Southeast University, Nanjing, Jiangsu 210009, P.R. China
\end{abstract}

Received June 28, 2019; Accepted November 21, 2019

DOI: $10.3892 / \mathrm{mmr} .2019 .10890$

\begin{abstract}
The present study investigated the role of cytochrome P450 family 2 subfamily E polypeptide 1 (CYP2E1) in the development and progression of gastric cancer (GC). The expression levels of CYP2E1 in MGC-803 GC cells and normal GES-1 cells were investigated via western blotting, and it was identified that the expression of CYP2E1 was different between GES-1 and MGC-803 cells. CYP2E1 was overexpressed in MGC-803 cells using a lentiviral vector GV358. Cell Counting Kit-8, flow cytometry, cell migration and Matrigel invasion assays suggested that overexpression of CYP2E1 promoted the proliferation and invasion, and inhibited the apoptosis of GC cells. The relationship between CYP2E1 expression and key signaling molecules in the PI3K/Akt/mTOR signaling pathway was assessed. Reverse transcription-quantitative PCR analysis showed that mTOR mRNA expression was significantly increased after overexpression of CYP2E1 $(\mathrm{P}<0.05)$. Western blotting results showed that overexpression of CYP2E1 upregulated the expression of phosphorylated (p)-Akt, p-mTOR and p-p70 ribosomal protein S6 kinase (P70S6K; Ser371) proteins $(\mathrm{P}<0.05)$. To further investigate the relationship between CYP2E1 and the PI3K/Akt/mTOR signaling pathway in GC cells, MGC-803 cells were treated with the PI3K inhibitor LY294002, and changes in the expression levels of PI3K, AKT, mTOR, P70S6K and CYP2E1 were observed. The present results showed that LY294002 downregulated the expression of PI3K, CYP2E1, AKT, mTOR and P70S6K $(\mathrm{P}<0.05)$. Therefore, changes in the biological
\end{abstract}

Correspondence to: Professor Xiao-Bing Shen, Key Laboratory of Environmental Medicine Engineering, Ministry of Education, School of Public Health, Southeast University, 87 Dingjiaqiao, Nanjing, Jiangsu 210009, P.R. China

E-mail: xb.shen@seu.edu.cn

Abbreviations: p-P70S6K, phosphorylated p70 ribosomal protein S6 kinase; CYP2E1, cytochrome P450 family 2 subfamily E polypeptide 1; RT-qPCR, reverse transcription-quantitative PCR

Key words: gastric cancer, CYP2E1, cytological function, PI3K/Akt/mTOR, LY294002 function of GC cells induced by CYP2E1 overexpression may be via the PI3K/Akt/mTOR signaling pathway.

\section{Introduction}

Gastric cancer (GC) is the 5th most commonly diagnosed cancer worldwide and the 3rd leading cause of cancer-related mortality, with $>1,000,000$ new cases and $\sim 783,000$ deaths in 2018 (equivalent to 1 death in every 12 cases worldwide) (1). Previous epidemiological studies have confirmed that the incidence of GC is affected by risk factors such as race, genetics, infectious agents, eating habits and other lifestyle habits (such as smoking and alcohol abuse) $(2,3)$. The development of genetics-based GC prevention and treatment strategies has become a hot topic in GC research. Although the liver is the main organ responsible for alcohol metabolism, a previous study demonstrated that the gastrointestinal tract is also involved in alcohol metabolism (4). Various enzymes in the gastrointestinal tract play a role in alcohol metabolism, including isoenzymes, cytochrome P450 (CYP) family 2 subfamily E polypeptide 1 (CYP2E1) and the catalase of alcohol dehydrogenase (5). A previous study revealed that alcohol abuse is a risk factor for cell damage and cancer, in which chronic ethanol intake can induce overexpression of CYP2E1 (6).

CYPs are a superfamily of proteins containing heme as a cofactor that play a crucial role in the metabolism of a diverse range of xenobiotics (7). $\mathrm{P} 450$ proteins play a critical role in chemical carcinogenesis by activating or inactivating carcinogens, which impacts the initiation and promotion of tumors (8). Previous studies have shown that CYP2E1 is not only significantly upregulated in the liver but it is also expressed in intestinal metaplasia, active inflammatory lesions in the stomach and endocrine cells in the gastric mucosa $(9,10)$. CYP2E1 activity can be activated by drugs and plants (isoniazid, Salvia miltiorrhiza, Schisandra chinensis), pollutants (phenylamine) and food ingredients (coffee and cocoa polyphenols) (11). Ethanol-induced inhibition of Akt phosphorylation and pharmacological regulation of Akt can lead to CYP2E1-induced hepatic oxidative stress, which could be a potential therapeutic strategy for ethanol-induced fatty liver (12). The effect of alcohol on CYP2E1 induction and the role of PI3K/Akt in protecting against the cytotoxicity 
of CYP2E1, suggest that reductions in the expression of key proteins in the PI3K signaling pathway may be a result of CYP2E1 overexpression (12). Taken together, these findings suggest that CYP2E1 serves as a crucial oncogene in human GC. However, a previous study focused on gene polymorphisms of CYP2E1 rather than the interaction between CYP2E1 and the PI3K/Akt/mTOR signaling pathway (13). The aim of the present study was to investigate the effects of CYP2E1 on the function of GC cells, and to explore the potential link between CYP2E1 and the PI3K/AKT/mTOR signaling pathway, therefore providing novel insight to facilitate the treatment and prevention of GC.

\section{Materials and methods}

Cell culture. Human gastric cancer cell MGC-803 and the normal gastric epithelial cell line GES-1 were purchased from Nanjing KeyGen Biotech Co., Ltd. The cells were cultured in RPMI-1640 medium with $10 \%$ FBS (both purchased from Thermo Fisher Scientific, Inc.), $100 \mu \mathrm{g} / \mathrm{ml}$ penicillin and $100 \mu \mathrm{g} / \mathrm{ml}$ streptomycin at $37^{\circ} \mathrm{C}$ in a humidified incubator at $5 \% \mathrm{CO}_{2}$. The PI3K inhibitor LY294002 (10 mmol/l; Cell Signaling Technology, Inc.) was dissolved in DMSO (cat. no. D2650; Sigma-Aldrich; Merck KGaA) and diluted with RPMI-1640 medium to $12.5,25$ and $50 \mu \mathrm{mol} / 1$. LY294002 was cultured with MGC-803 cells at $37^{\circ} \mathrm{C}$ for $24 \mathrm{~h}$ in a humidified incubator at $5 \% \mathrm{CO}_{2}$.

Plasmid construction and transfection of GC cells. The CYP2E1 construct was synthesized and integrated into a recombinant lentiviral vector GV358 (Shanghai GeneChemCo., Ltd.). CYP2E1 overexpression was accomplished with the recombinant lentiviral vector (Shanghai GeneChem Co., Ltd.), and the empty vector was used as a control. MGC-803 cells were seeded into 96 -well plates $\left(5 \times 10^{3}\right.$ cells/well in $100 \mu$ l complete medium), and three infection gradients ( $\mathrm{MOI}=10, \mathrm{MOI}=20$, $\mathrm{MOI}=30$ ) were used to infect the cells using FuGENE ${ }^{\circledR} 6$ transfection reagent (Nanjing KeyGen Biotech Co., Ltd). The MGC-803 cells were incubated for 24 and $48 \mathrm{~h}$ after transfection for subsequent experiments.

$R N A$ isolation and reverse transcription-quantitative (RT-q) $P C R$ analysis. The expression levels of CYP2E1 and the normalization gene $\beta$-actin were assessed via RT-qPCR. Total RNA was extracted from cells using TRIzol ${ }^{\circledR}$ reagent (Invitrogen; Thermo Fisher Scientific, Inc.), and RNA purity was detected with a NanoDrop ${ }^{\mathrm{TM}} 2000$ spectrometer (NanoDrop Technologies; Thermo Fisher Scientific, Inc.). In accordance with the manufacturer's protocol, RNA $(1 \mu \mathrm{g})$ was reverse transcribed into cDNA via a two-step reverse transcription reaction performed $\left(37^{\circ} \mathrm{C}\right.$ for $15 \mathrm{~min}$ and $85^{\circ} \mathrm{C}$ for $5 \mathrm{sec}$ ) using a Takara cDNA Synthesis Kit (Thermo Fisher Scientific, Inc.). RT-qPCR was performed with a StepOnePlus real-time PCR system (Applied Biosystems; Thermo Fisher Scientific, Inc.) and SYBR Green One-Step Real-Time RT-PCR Master Mixes (Thermo Fisher Scientific, Inc.) to measure the expression level of the selected long non-coding RNA. Amplifications were performed with 2 min enzyme activation at $95^{\circ} \mathrm{C}$, followed by 40 cycles of denaturation at $95^{\circ} \mathrm{C}$ for $15 \mathrm{sec}$, and then annealing/extension at $60^{\circ} \mathrm{C}$ for
$15 \mathrm{sec}$. At the end of each run, melting curve analysis was performed at $72^{\circ} \mathrm{C}$ for $30 \mathrm{sec}$. The comparative $\mathrm{Cq}$ method was used to measure the relative fold change in CYP2E1 levels in GC cells. Relative quantitation values of mRNA expressions were calculated using the $2^{-\Delta \Delta \mathrm{Cq}}$ method (14). The primers were obtained from Kingsley Biotechnology Co., Ltd. The mRNA primer sequences for CYP2E1 and the housekeeping gene $\beta$-actin were as follows: CYP2E1 forward, 5'-GCCATCAAG GATAGGCAAGA-3' and reverse, 5'-TCCAGAGTTGGCACT ACGACT-3'; $\beta$-actin forward, 5'-TCACCCACACTGTGCCCA TCTACGA-3' and reverse, 5'-CAGCGGAACCGCTCATTG CCAATGG-3'.

Western blot analysis. Total protein was extracted from cells using RIPA lysate reagent (Thermo Fisher Scientific, Inc.), and the total protein concentration was detected by a BCA protein detection kit (Thermo Fisher Scientific, Inc.) Total protein $(20 \mu \mathrm{g} /$ lane) were separated using SDS-PAGE (10\%) and then transferred to a nitrocellulose membrane. The membrane was blocked in TBS-Tween-20 (TBST) with $5 \%$ milk at room temperature for $2 \mathrm{~h}$ and then incubated with primary antibody in 5\% BSA-TBST $(1: 1,000)$ overnight at $4^{\circ} \mathrm{C}$. Then, the membrane was washed with TBST and incubated with secondary antibody $(1: 10,000)$ at room temperature for $1 \mathrm{~h}$. Protein bands were detected with an ECL chromogenic kit (Thermo Fisher Scientific, Inc.). Images were captured using a Tanon-5200 chemiluminescence imaging system (Tanon Science and Technology Co.,Ltd.), and ImageJ software (v1.8.0; National Institutes of Health) was used for densitometric analysis. Specific primary antibodies against $\beta$-actin (cat.no. 4967S), PI3K (cat. no. 4292S), phosphorylated (p)-PI3K (cat. no. 4228T), Akt (cat. no. 9272S), p-Akt (cat. no. 9271T), mTOR (cat. no. 2972S), p-mTOR (cat. no. 2971S), p70 ribosomal protein S6 kinase (P70S6K; cat. no. 9202S), p-P70S6K (Ser371; cat. no. 9204S) and CYP2E1 (cat. no. ab28146; Abcam) and the secondary antibody (anti-rabbit IgG, horseradish peroxidase-conjugated antibody; cat. no. 7074P2) was purchased from Cell Signaling Technology, Inc. The high molecular weight protein marker was supplied by Bio-Rad Laboratories, Inc., and the small molecular weight protein marker was supplied by Thermo Fisher Scientific, Inc.

Cell proliferation assay. The proliferation ability of CYP2E1-overexpressing MGC-803 cells was determined with a Cell Counting Kit-8 (CCK-8; Yisheng Biotechnology Co., Ltd.). In line with the manufacturer's protocol, cells were seeded into 96 -well plates $\left(5 \times 10^{3}\right.$ cells/well in $100 \mu 1$ complete medium) in a $\mathrm{CO}_{2}$ incubator at $37^{\circ} \mathrm{C}$ for $6,12,24,36,48$ and $60 \mathrm{~h}$, followed by addition of $10 \mu \mathrm{l}$ of the CCK- 8 mixture to each well. After incubation for $2 \mathrm{~h}$ at $37^{\circ} \mathrm{C}$ in an incubator, the absorbance was measured at a wavelength of $450 \mathrm{~nm}$ using a microplate reader (Gene Company Ltd.).

Cell apoptosis assay. For flow cytometry analysis, cells were seeded on 6 -well plates at $5 \times 10^{5}$ cells/well, with three replicate wells per experimental group. After a $48 \mathrm{~h}$ culture period at $37^{\circ} \mathrm{C}$, the cells were harvested and washed twice with phosphate buffer, resuspended in $300 \mu \mathrm{l}$ of binding buffer, and reacted with $5 \mu \mathrm{l}$ of Annexin V-allophycocyanin (APC) and $5 \mu \mathrm{l}$ of hypotonic propidium iodide (PI) solution for $10 \mathrm{~min}$ 

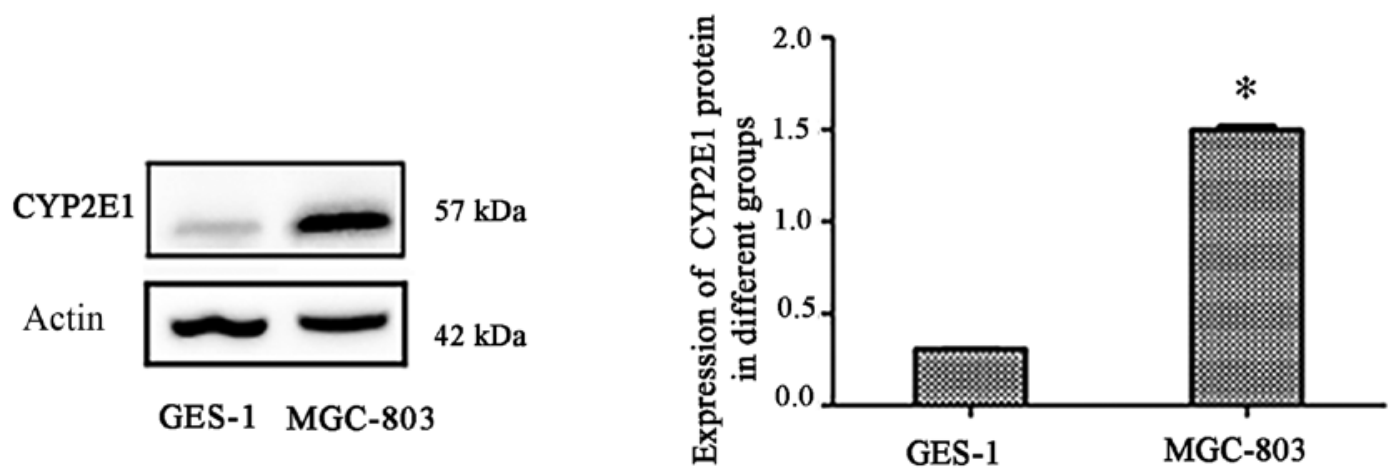

Figure 1. CYP2E1 protein expression in GES-1 and MGC-803 cells. "P<0.05. CYP2E1, cytochrome P450 family 2 subfamily E polypeptide 1.

at room temperature. Then, $300 \mu 1$ binding buffer solution was added and the cells were examined via flow cytometry for $10 \mathrm{~min}$. The cells were analyzed using a BD flow cytometer (BD Via; BD Biosciences) (15). FlowJo software (v10.0; $\mathrm{BD}$ Biosciences) was used to analyze these data. The apoptosis rate was calculated as the percentage of early + late apoptotic cells. An Annexin V-APC/PI apoptosis kit (MultiSciences Biotech, Co., Ltd.) was used for the cell apoptosis assay.

Cell migration assay. Cell migration was assayed using Transwell inserts (Corning Inc.). MGC-803 cells transduced for $96 \mathrm{~h}$ were collected and washed three times with PBS. MGC-803 CYP2E1 cells were resuspended in RPMI-1640 medium containing 1\% FBS (Thermo Fisher Scientific, Inc.) and inoculated into the upper Transwell chamber at a density of $5 \times 10^{4}$ cells/well (hole, $8 \mu \mathrm{m}$ ). Next, RPMI-1640 medium containing 10\% FBS was added to the lower Transwell chamber, and the cells were cultured at $37^{\circ} \mathrm{C}$ in a humidified incubator containing $5 \% \mathrm{CO}_{2}$. The chamber was removed after 24-h incubation. The cells were fixed for $10 \mathrm{~min}$ at room temperature with $95 \%$ ethanol, stained with $0.1 \%$ crystal violet for $10 \mathrm{~min}$ at room temperature, and then washed with PBS. The chamber was air-dried, and 5-10 fields in each chamber were randomly selected for cell counts. Images were acquired for later analyses. The number of cells that passed through the chamber was compared between the MGC-803 CYP2E1 and MGC-803 NC groups. Visualization was performed with an FSX100 biological imaging system (magnification, x500; Olympus Corporation), and the images were analyzed using Image-Pro Plus 6.0 software (Media Cybernetics, Inc.).

Matrigel invasion assay. The invasive ability of MGC- 803 CYP2E1 and MGC-803 NC cells was examined with Matrigel (Matrigel Gel and serum-free medium preparation, 1:8) at $4^{\circ} \mathrm{C}$ overnight using a Transwell package. MGC-803 cells transduced for $24 \mathrm{~h}$ were collected and washed three times with PBS. MGC-803 CYP2E1 cells were resuspended in RPMI-1640 medium containing $1 \%$ FBS and seeded at a density of $1-5 \times 10^{5}$ cells/well into the top of the chamber (pores, $8-\mu \mathrm{m}$; the chamber was removed from $4^{\circ} \mathrm{C}$ before use and placed at $37^{\circ} \mathrm{C}$ for $1 \mathrm{~h}$ to coagulate the matrix). Medium containing 10\% FBS was added to the bottom of the Transwell chamber. After 24-h incubation at $37^{\circ} \mathrm{C}$, the remaining cells in the upper chamber were scraped away using cotton swabs. The invasive cells were fixed with $95 \%$ ethanol for $10 \mathrm{~min}$ at room temperature, stained with $0.1 \%$ crystal violet for $10 \mathrm{~min}$ at room temperature, and then washed with PBS. The chamber was air dried and imaged with an FSX100 Bio Imaging Navigator (magnification, x500; Olympus Corporation), and the number of cells that penetrated the chambers was determined as described above for the cell migration assay.

Statistical analysis. The statistical significance of the RT-qPCR, protein expression, flow cytometry, migration and invasion assay results were analyzed using GraphPad Prism 8.0 (GraphPad Software, Inc.). Data are expressed as the mean \pm SD. Student's t-test and one-way ANOVA followed by Dunnett's test were used to assess the statistical significance of differences. $\mathrm{P}<0.05$ was considered to indicate a statistically significant difference.

\section{Results}

CYP2E1 expression in GC cells. Western blot analysis showed that the protein expression level of CYP2E1 in MGC-803 cells was significantly higher than in GES-1 cells $(\mathrm{P}<0.05$; Fig. 1). These results suggested that the expression of CYP2E1 is different between GES-1 and MGC-803.

Lentiviral-mediated infection to induce CYP2E1 overexpression in GC cells in vitro. To further explore the biological function of CYP2E1, a CYP2E1 lentiviral vector was constructed to induce CYP2E1 overexpression. MGC-803 cells were infected with a CYP2E1 lentiviral vector or the empty vector for 24-72 h. MGC-803 cells were efficiently infected with the lentivirus-CYP2E1. RT-qPCR results (Fig. 2A) indicated that the CYP2E1 expression level in MGC-803 cells transduced with the lentivirus-CYP2E1 overexpression vector was significantly increased compared with the MGC-803 NC cells $(63$-fold; $\mathrm{P}<0.05)$. Western blotting showed that protein expression level of CYP2E1 was significantly increased in the MGC-803 cells transduced with the lentivirus-CYP2E1 overexpression vector compared with the MGC-803 NC group ( $\mathrm{P}<0.05$; Fig. 2B).

Overexpression of CYP2E1 affects the proliferation and apoptosis of GC cells in vitro. To determine whether CYP2E1 affects GC cell proliferation and apoptosis, a CCK-8 cell 


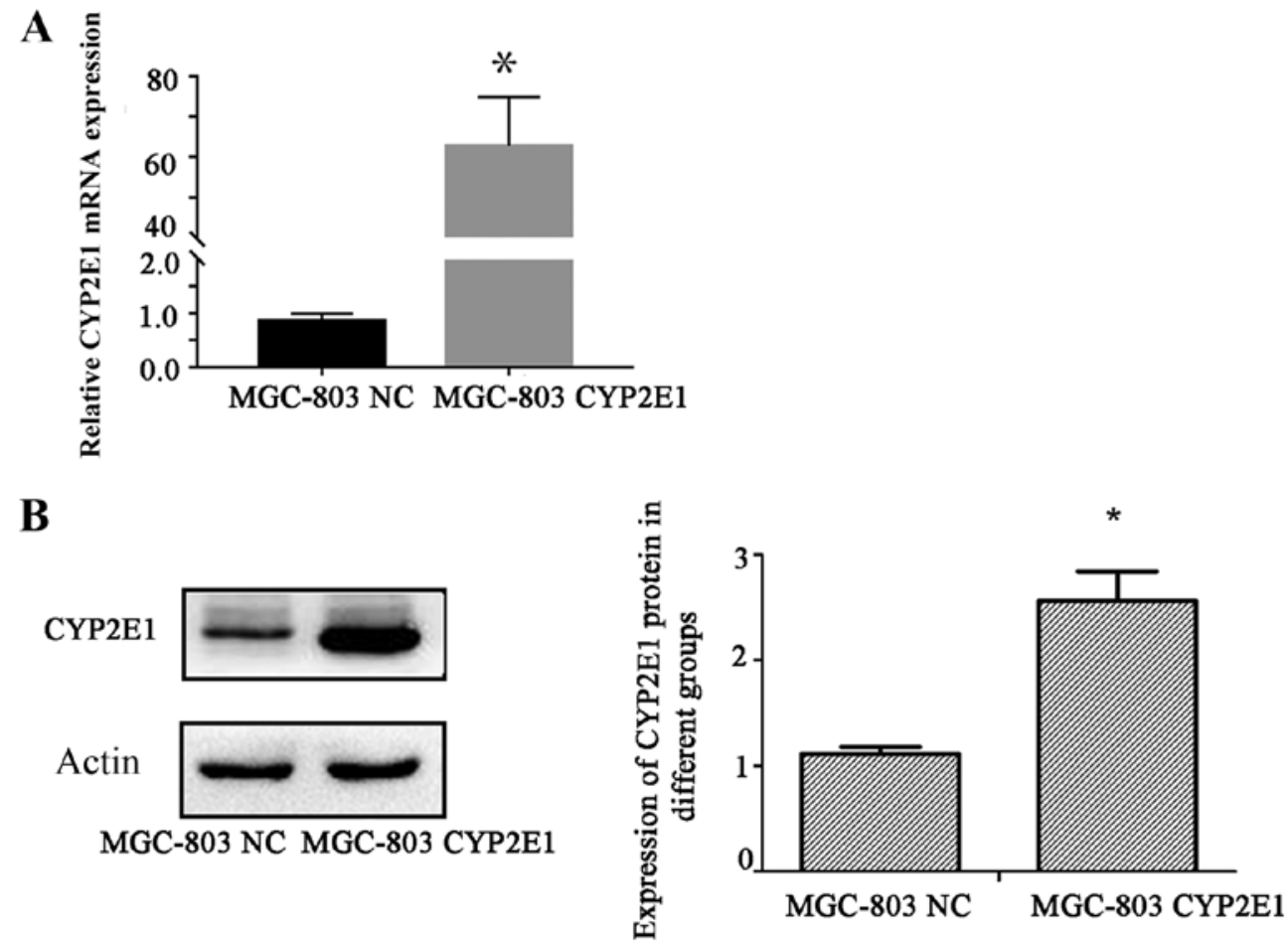

Figure 2. Overexpression of CYP2E1 in gastric cancer cells. (A) Reverse transcription-quantitative PCR detected MGC-803 lentivirus-mediated CYP2E1 overexpression. (B) Western blot analysis detected MGC-803 lentivirus-mediated CYP2E1 overexpression. * $\mathrm{P}<0.05$ vs. MGC-803 NC. CYP2E1, cytochrome P450 family 2 subfamily E polypeptide 1; NC, negative control.

proliferation assay and flow cytometry were used to test the proliferative capacity and apoptotic rate of MGC-803 cells overexpressing CYP2E1.

In MGC-803 CYP2E1 cells, cell growth increased from $12 \mathrm{~h}$ and was time-dependent compared with negative control cells $(\mathrm{P}<0.05$; Fig. 3A). Annexin V-APC/PI staining combined with flow cytometry showed that the apoptotic rate in the MGC-803 NC group was significantly higher than that in the MGC-803 CYP2E1 group at $24 \mathrm{~h}(\mathrm{P}<0.05$; Fig. 3B and $\mathrm{C})$.

CYP2E1 overexpression promotes the migration and invasion of GC cells. In addition, it was investigated whether CYP2E1 plays a critical role in migration and invasion of GC cells. A Transwell assay was used to analyze the invasive and migratory abilities of GC cells after CYP2E1 gene overexpression. The migratory ability of MGC- 803 cells was significantly increased after transduction with the lenti-CYP2E1-overexpression plasmid compared with the negative control (Fig. 4A and B). Similarly, the Matrigel invasion assay results indicated that the invasive ability of MGC-803 cells was significantly increased after transduction with the lenti-CYP2E1-vector $(\mathrm{P}<0.05$; Fig. 4C and D).

CYP2E1 regulates key PI3K/Akt/mTOR signaling proteins. $\mathrm{PI} 3 \mathrm{~K} / \mathrm{Akt} / \mathrm{mTOR}$ is a crucial signaling pathway involved in the carcinogenicity and tumorigenesis of different cancer types, and is closely related to cell biological functions, such as energy metabolism, proliferation, apoptosis and cell invasion (16-19). Previous studies have revealed that certain mRNAs are co-expressed with CYP2E1 and participate in the $\mathrm{PI} 3 \mathrm{~K} / \mathrm{Akt} / \mathrm{mTOR}$ signaling pathway $(20,21)$. Therefore, the present study investigated whether CYP2E1 was involved in the regulation of GC cell proliferation, apoptosis and invasion via $\mathrm{PI} 3 \mathrm{~K} / \mathrm{Akt} / \mathrm{mTOR}$ signaling proteins. mRNAs and proteins were isolated from lysed MGC-803 cells that were transduced with CYP2E1 overexpression lentivirus or the negative control. RT-qPCR results revealed that the expression level of mTOR mRNA in MGC-803 cells transduced with the lentiviral-CYP2E1 overexpression vector was significantly higher compared with the MGC-803 NC group ( $\mathrm{P}<0.05$; Fig. 5A). However, no significant differences in PI3K and Akt mRNA expression levels were observed between MGC-803 CYP2E1 cells and MGC-803 NC cells. Western blotting results revealed that CYP2E1 overexpression increased the relative expression levels of p-Akt, p-mTOR, and p-P70S6KSer371 compared with those in the negative controls $(\mathrm{P}<0.05$; Fig. $5 \mathrm{~B})$. Therefore, it is possible that CYP2E1 is involved in the malignant progression of GC cells via p-Akt, p-mTOR and p-P70S6KSer371 proteins in the PI3K/Akt/mTOR signaling pathway.

Effect of PI3K/Akt/mTOR signaling pathway inhibitor on CYP2E1 expression in MGC-803 cells. To further explore the role of CYP2E1 in MGC-803 cells, the PI3K/Akt/mTOR signaling pathway was inhibited using the PI3K inhibitor LY294002. In the LY294002-treated group, PI3K, Akt, mTOR, p70S6K and CYP2E1 protein expression levels decreased with increasing inhibitor concentration compared with the control group (Fig. 6; $\mathrm{P}<0.05$ ). These results suggested that CYP2E1 could function as an oncogene in GC by participating in the PI3K/Akt/mTOR signaling pathway. CYP2E1 protein expression may be mediated by the PI3K signaling pathway and key downstream mediators. 

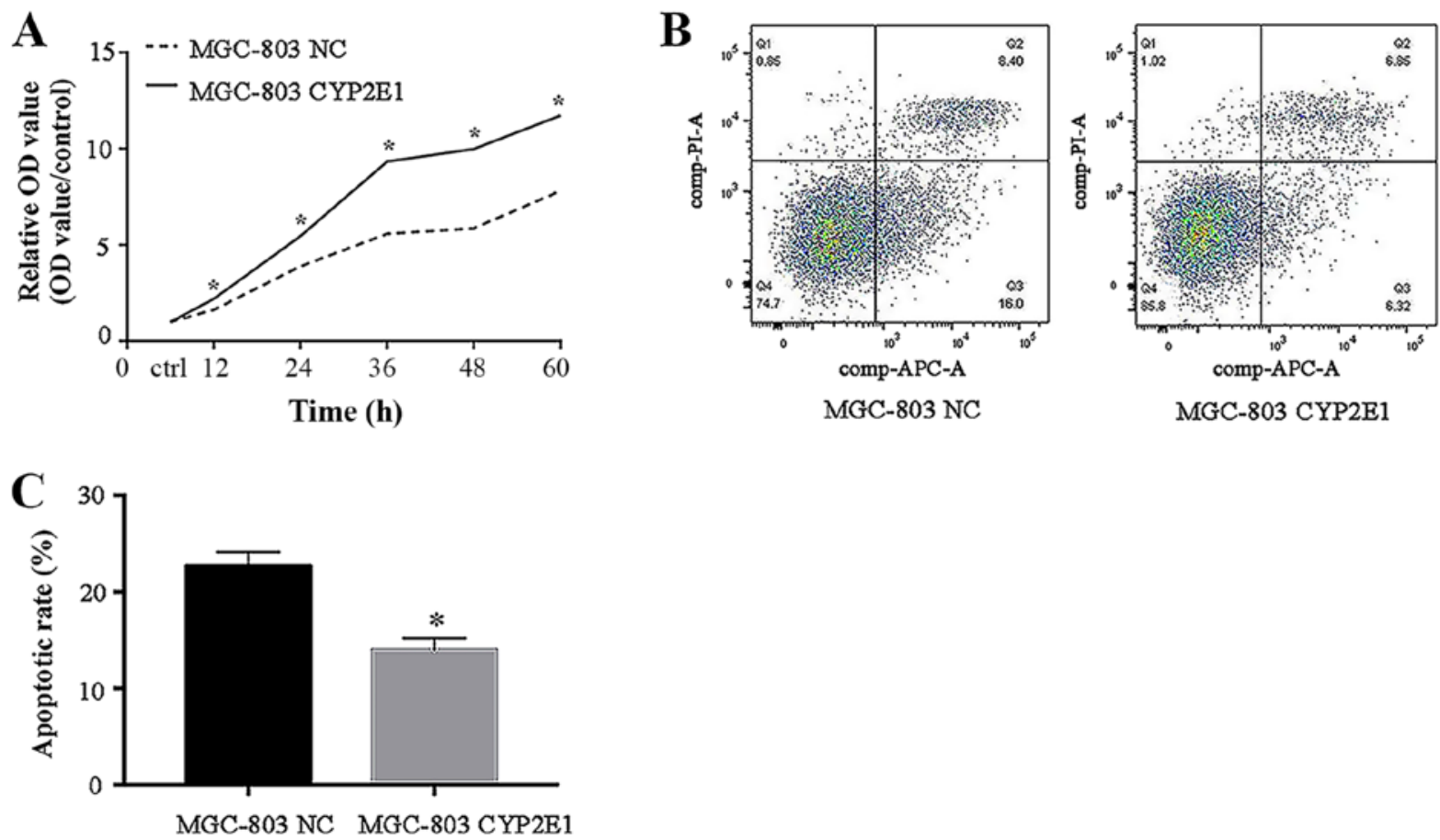

Figure 3. Overexpression of CYP2E1 affects the proliferation and apoptosis of gastric cancer cells in vitro. (A) Cell proliferation was measured using a Cell Counting Kit-8 assay. (B) Flow cytometry apoptosis assay results. (C) Apoptotic rate of transfected CYP2E1 overexpressing MGC-803 cells compared with the corresponding NC. Data are presented as the mean \pm SD. "P $<0.05$ vs. MGC- 803 NC. APC, allophycocyanin; ctrl, control; CYP2E1, cytochrome P450 family 2 subfamily E polypeptide 1; NC, negative control; PI, propidium iodide.

A

MGC-803 NC

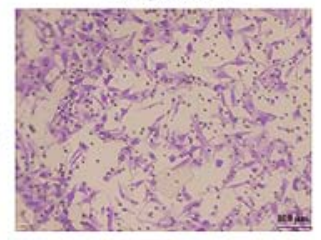

MGC-803 CYP2E1

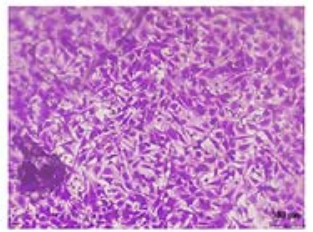

C

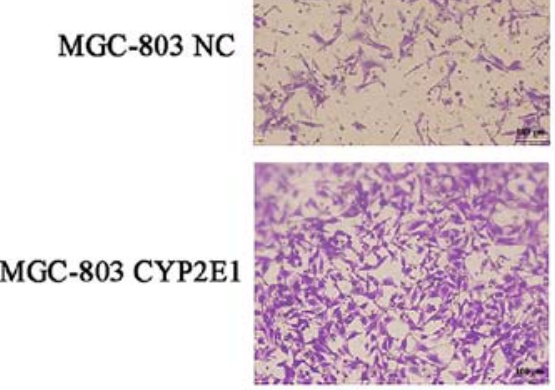

B

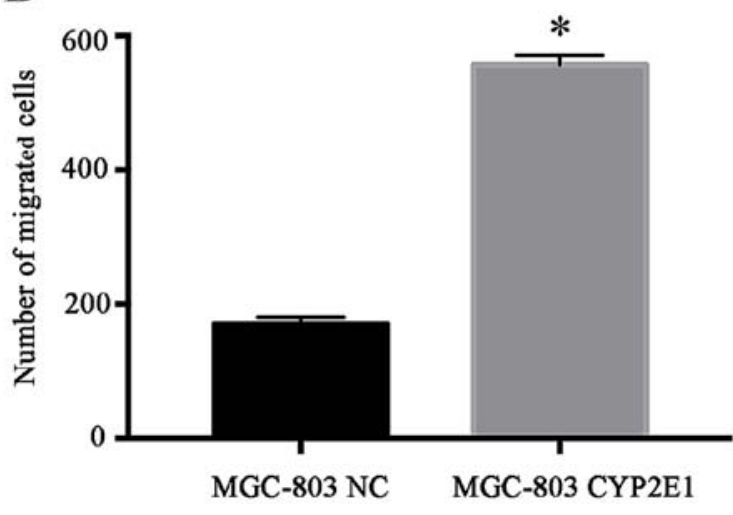

D

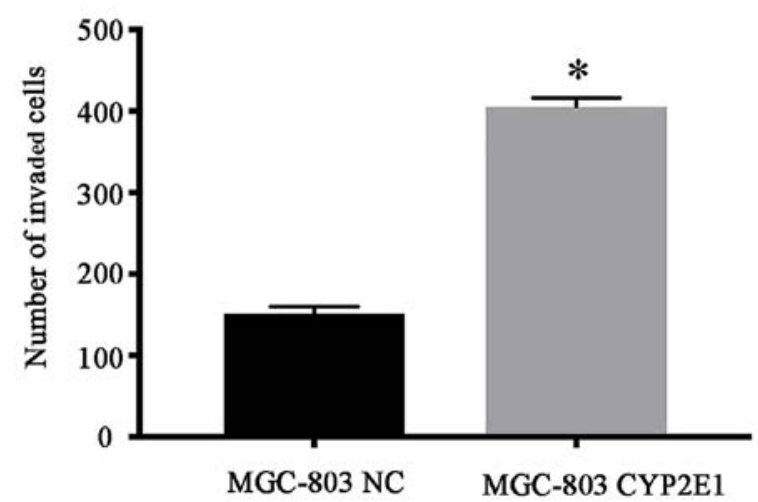

Figure 4. CYP2E1 overexpression promotes the migration and invasion of gastric cancer cells. (A) Representative images of MGC-803 cells with CYP2E1 overexpression from Transwell migration assay. (B) Number of migrated cells presented as mean \pm SD. (C) Representative images of MGC-803 CYP2E1 overexpressing cells in Matrigel invasion assay. (D) Number of invaded cells presented as mean \pm SD. Scale bars, $100 \mu \mathrm{m}$. ${ }^{*} \mathrm{P}<0.05$ vs. MGC- $803 \mathrm{NC}$. CYP2E1, cytochrome P450 family 2 subfamily E polypeptide 1; NC, negative control. 

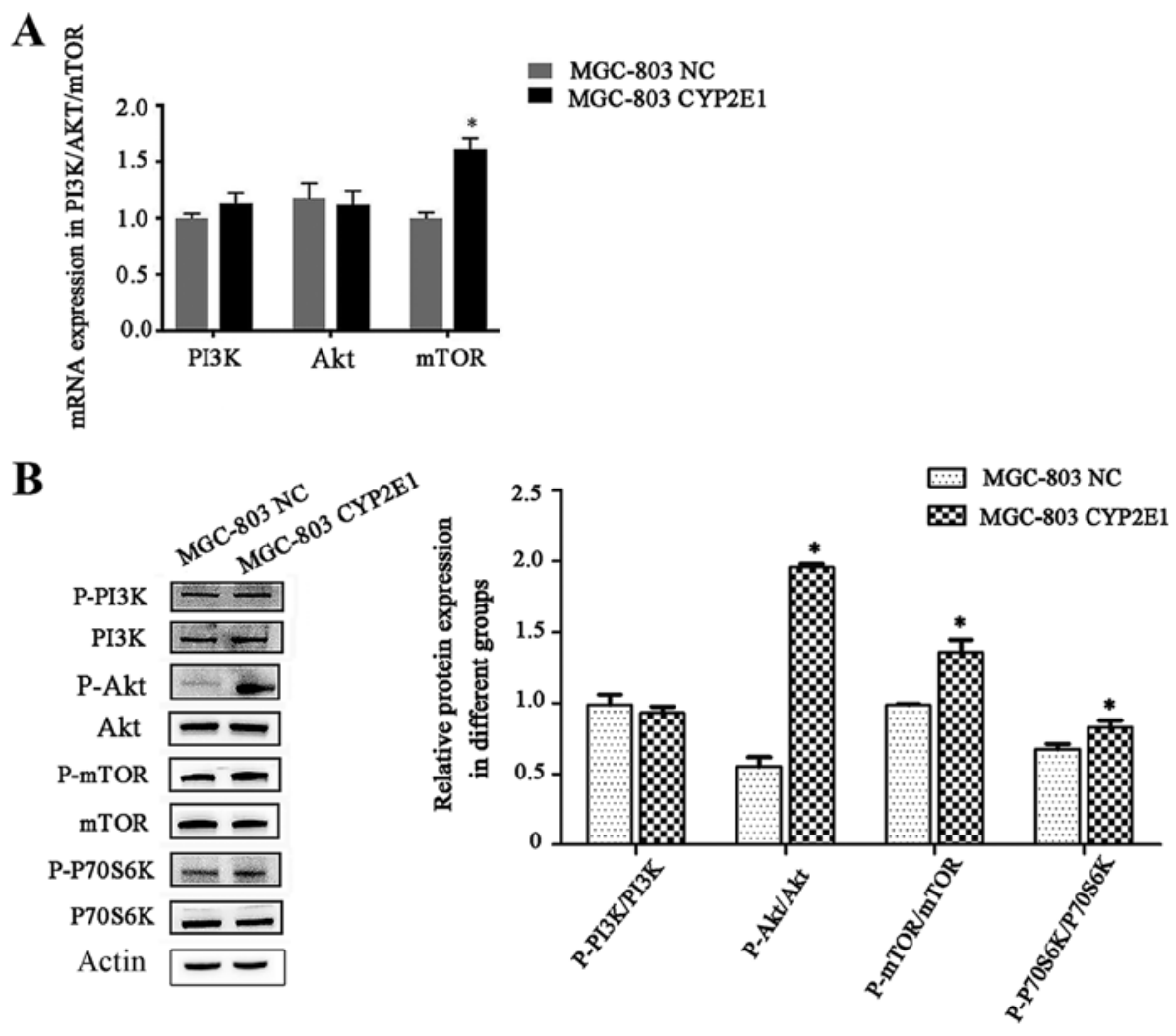

Figure 5. CYP2E1 regulates key proteins of the PI3K/Akt/mTOR signaling pathway. (A) Reverse transcription-quantitative PCR analysis indicated that CYP2E1 overexpression regulated the mRNA expression levels of key genes in the PI3K/Akt/mTOR signaling pathway. (B) Western blot analysis results indicated that CYP2E1 overexpression regulated key proteins of the PI3K/Akt/mTOR signaling pathway. "P<0.05 vs. MGC-803 NC. CYP2E1, cytochrome P450 family 2 subfamily E polypeptide 1; P70S6K, p70 ribosomal protein S6 kinase; NC, negative control; p-, phosphorylated.
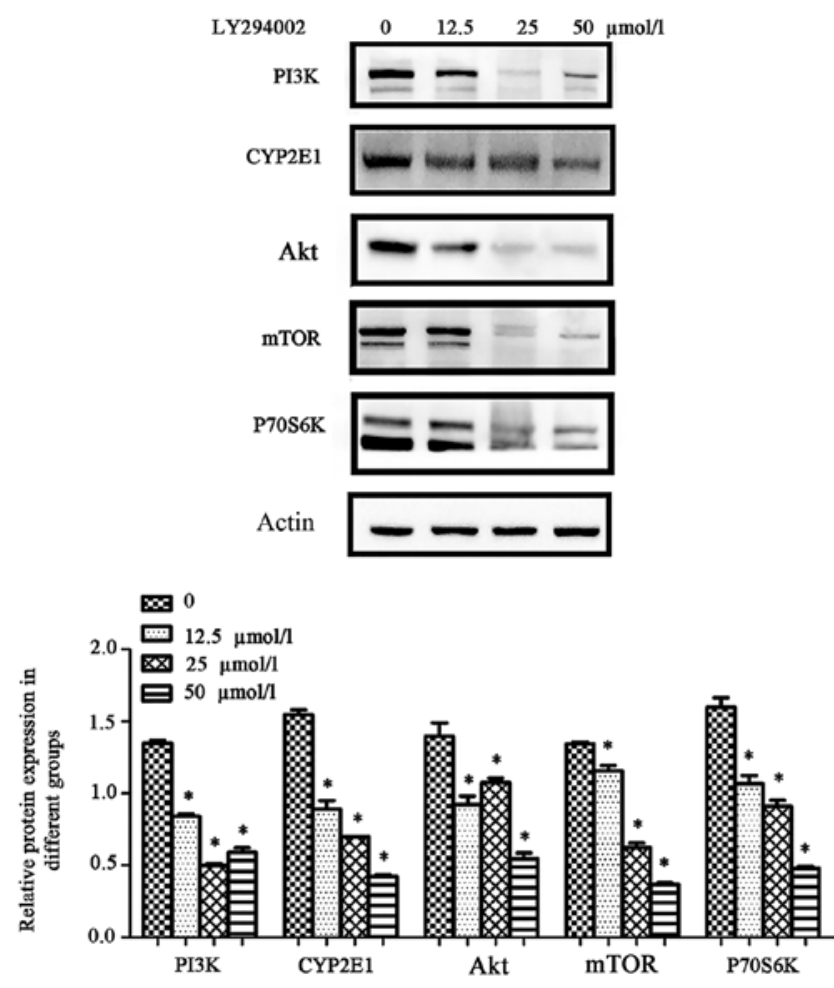

Figure 6. Effect of PI3K/Akt/mTOR signaling pathway inhibitor on CYP2E1 expression in MGC-803 cells. Protein expression of key signaling molecules in the PI3K/Akt/mTOR signaling pathway and CYP2E1 expression level after treatment with differentconcentrations of the PI3K inhibitorLY294002. $\mathrm{P}<0.05$ vs. $0 \mu \mathrm{mol} / 1$. CYP2E1, cytochrome P450 family 2 subfamily E polypeptide 1; P70S6K, p70 ribosomal protein S6 kinase.

\section{Discussion}

Previous studies have shown that the CYP2E1 can promote the metabolism of proto-oncogenes, and its differential expression may play a part in tumor maintenance, evolution and metastasis (22-24). CYP2E1 protein activates a large number of low molecular weight xenobiotics, which are involved in the development of alcohol-related GC by activating proto-oncogenes $(25,26)$. However, there are few studies on the specific role of CYP2E1 in the development and progression of GC.

Therefore, the present study compared the protein expression levels of CYP2E1 in MGC-803 and GES-1 cells to explore the possible role and activation of CYP2E1 in GC. The present results suggested that the expression of CYP2E1 is differentially expressed between GES-1 and MGC- 803 cells. A previous study demonstrated that CYP2E1 is primarily expressed in the liver, lung and kidney, while low expression is found in the stomach (27). Another study showed that alcohol-induced CYP2E1 protein expression is primarily increased via the inhibition of CYP2E1 protein degradation and the enhancement of the structural stability of CYP2E1 protein (28). The differential expression level of CYP2E1 in tumor cells may be used as a marker to assess tumor progression and to determine the factors related to the differential regulation of gene expression.

To further study the possible regulatory mechanism of CYP2E1 on the biological features of GC cells, MGC-803 cells transduced with the CYP2E1 lentiviral vector that induces CYP2E1 overexpression were used. The CYP2E1 mRNA 
expression increase in CYP2E1-overexpressing MGC-803 cells was higher than the protein level increase. Therefore, CYP2E1 gene expression may be non-linearly related to transcriptional translation.

The effect of CYP2E1 on the function of GC cells was investigated. CCK- 8 cell proliferation assay and flow cytometry results both indicated that overexpression of CYP2E1 led to a significant increase in MGC-803 cell proliferation and inhibition of apoptosis. Apoptosis has a large impact on slowing cell hyperproliferation, and the PI3K/Akt/mTOR signaling pathway is involved in multiple antiapoptotic pathways $(17,29)$. A previous study demonstrated that high CYP1B1 expression may cause changes in the response of tumor cells to drugs, and affect the metabolism or activation of environmental carcinogens in breast cancer, thereby enhancing the malignancy of cancer (30). Another previous study indicated that chronic CYP2E1 overexpression activated the mitogen-activated protein kinase pathway, which led to an increase in hepatocellular carcinoma cell survival and protected against menadione-induced apoptosis (31). Metastasis is the leading cause of gastric cancer-related death, and elucidating the signaling pathways involved in the metastatic cascade has become a key goal in the development of new and effective treatments aimed at reducing GC mortality (32). Therefore, the present study investigated whether CYP2E1 affects the invasive and migratory abilities of GC cells, using MGC-803 cells transduced with a lentiviral CYP2E1 overexpression vector. The present results showed that the invasive and migratory abilities of MGC- 803 cells were significantly increased after stable transduction with the lenti-CYP2E1 overexpression vector, which suggested that CYP2E1 overexpression may induce the migration and invasion of GC cells. Previous studies have shown that increased oxidative stress induced by CYP2E1 can damage cellular and mitochondrial components, and accelerate cancer progression $(33,34)$.

Abnormal activation of the PI3K/Akt/mTOR signaling pathway is closely related to cell functional features, such as cancer occurrence, progression, invasion and metastasis (35). The PI3K/Akt/mTOR pathway is inactive in normal tissues but confers a tumor cell growth advantage in cancer (36). Therefore, the present study explored the association between the PI3K/Akt/mTOR signaling pathway and CYP2E1 based on functional changes in GC cells. RT-qPCR results showed that the mRNA expression level of mTOR increased following overexpression of CYP2E1, suggesting that the functional changes in GC cells induced by overexpression of CYP2E1 may be related to downstream molecules in the PI3K/Akt/mTOR signaling pathway. Western blotting was used to detect the expression of proteins in the PI3K/Akt/mTOR signaling pathway. Western blotting results revealed that CYP2E1 overexpression increased the expression levels of p-Akt, p-mTOR, and p-P70S6KSer371 compared with the negative controls. The expression level of p-Akt increased in MGC-803 cells overexpressing CYP2E1, suggesting that CYP2E1 may play a role in modulating Akt. p-Akt can disrupt the apoptotic protein Bad, and inhibit the formation of the protein complex and apoptosis of the cells (37). A previous study showed that p-Akt reduces E-cadherin and intercellular adhesion by activating glycogen synthase kinase 3 (GSK3), the downstream factors of PI3K/Akt signaling pathway, which increases tumor cell migration and invasion potential (38). In the present study, overexpression of CYP2E1 may decrease apoptosis, and promote the invasion and migration of GC cells. The malignant changes of GC cells may be related to the activation of Akt (39). The increased expression of mTOR mRNA and phosphorylation of mTOR protein suggested that overexpression of CYP2E1 increases mTOR transcription and activation. mTOR activation is primarily caused by increased mTOR phosphorylation, and as a result p-mTOR protein expression level can reflect the activation level of the PI3K/Akt/mTOR signaling pathway in cells $(40,41)$. A previous study showed that the expression level of p-mTOR in normal tissues was significantly lower than in gastric cancer tissues (34). The expression of p-mTOR was positively associated with TNM stage, depth of invasion and lymph node involvement in gastric cancer (42). In the present study, the phosphorylation level of P70S6K increased in MGC-803 cells overexpressing CYP2E1, indicating that CYP2E1 modulated mTOR and its downstream effector P70S6K. One of the phosphorylated substrates that activates mTOR could be P70S6K. It has been previously shown that P70S6K1 controls cell growth by increasing mRNA translation, while p-P70S6K promotes remodeling of actin filaments, as well as cell migration, tumor invasion and metastasis (43). Another previous study has demonstrated that the inhibition of CYP2E1 can reverse the decrease of GSK-3 $\beta$ phosphorylation downstream of the PI3K/Akt/mTOR signaling pathway induced by chronic ethanol (13). Additionally, it has been shown that the expression of CYP2E1 decreases with increasing insulin concentration, and has been confirmed to be regulated by the PI3K/Akt/mTOR signaling pathway (44). Therefore, overexpression of CYP2E1 may be able to activate the PI3K/Akt/mTOR signaling pathway in GC and regulate the biological function of GC cells via changes to Akt, mTOR and key downstream factors in the signaling pathway.

To further investigate the possible functional site of CYP2E1 in the PI3K/Akt/mTOR signaling pathway, targeted inhibitors of the PI3K/Akt/mTOR signaling pathway were used. Unlike wortmannin, the pharmacological inhibitor LY294002 is a synthetic quercetin compound that is a specific inhibitor of PI3K and capable of inhibiting PI3K-associated proteins (45). In the present study, LY294002 inhibited the expression of PI3K, CYP2E1, Akt, mTOR and P70S6K protein in MGC-803 cells in a dose-dependent manner. LY294002 may regulate the expression of downstream signal molecules and CYP2E1 by affecting the expression of key signal molecules in the upstream signaling pathway. Therefore, the present study hypothesized that the functional site of CYP2E1 may be midstream in the P13K/Akt/mTOR signaling pathway.

The present results may have potential clinical implications, but limitations of the study must be addressed. The effect of a single gene on the malignancy of tumor biology is influenced by a variety of uncertainties. Therefore, it is necessary to further explore the specific functions and molecular mechanisms of CYP2E1 via in vivo and in vitro experiments.

In conclusion, the present results suggested an oncogenic role of CYP2E1 in GC. CYP2E1 could affect the biological function of GC cells through the PI3K/Akt/mTOR signaling pathway. CYP2E1 was proposed to promote the proliferation of MGC- 803 cells, inhibit apoptosis and enhance the migration and invasion of GC cells by activating the PI3K/Akt/mTOR signaling pathway. LY294002 was found to reduce the 
activation of key signaling molecules and CYP2E1 protein expression level in GC cells.

\section{Acknowledgements}

The authors would like to thank Dr Tong Liu (Southeast University, Nanjing) who provided technical assistance in the study.

\section{Funding}

The present study was supported by The National Natural Science Foundation of China (grant no. 81472940).

\section{Availability of data and materials}

The datasets used and/or analyzed during the current study are available from the corresponding author on reasonable request.

\section{Authors' contributions}

RYW and XBS contributed to the design of the study protocol. XWC, WWZ, FJ and MQL performed statistical analysis and experiments. RYW contributed to the writing of the study protocol. All authors approved the final version of the manuscript.

\section{Ethics approval and consent to participate}

Not applicable.

\section{Patient consent for publication}

Not applicable.

\section{Competing interests}

The authors declare that they have no competing interests.

\section{References}

1. Bray F, Ferlay J, Soerjomataram I, Siegel RL, Torre LA and Jemal A: Global cancer statistics 2018: GLOBOCAN estimates of incidence and mortality worldwide for 36 cancers in 185 countries. CA Cancer J Clin 68: 394-424, 2018.

2. Kankeu Fonkoua L and Yee NS: Molecular characterization of gastric carcinoma: Therapeutic implications for biomarkers and targets. Biomedicines 6: E32, 2018.

3. Itoga $S$, Harada $S$ and Nomura F: Polymorphism of the 5'-flanking region of the CYP2E1 gene: An association study with alcoholism. Alcohol Clin Exp Res 25 (Suppl 6): S11-S55, 2006.

4. Kaibara N, Kobori A, Sekime A and Miyasaka K: The menstrual cycle influences the gastric emptying of alcohol. Biomed Res 36: 411-415, 2015

5. Zhu H, Jia Z, Misra H and Li YR: Oxidative stress and redox signaling mechanisms of alcoholic liver disease: Updated experimental and clinical evidence. J Dig Dis 13: 133-142, 2012.

6. Jin M, Kumar A, Kumar S and Srivastava RK: Ethanol-mediated regulation of cytochrome $\mathrm{P} 4502 \mathrm{~A} 6$ expression in monocytes: Role of oxidative stress-mediated $\mathrm{PKC} / \mathrm{MEK} / \mathrm{Nrf} 2$ pathway. PLoS One 7: e35505, 2012.

7. Ortega Ugalde S, Boot M, Commandeur JNM, Jennings $\mathrm{P}$, Bitter W and Vos JC: Function, essentiality, and expression of cytochrome P450 enzymes and their cognate redox partners in Mycobacterium tuberculosis: Are they drug targets? Appl Microbiol Biotechnol 103: 3597-3614, 2019.
8. Qu R, Li X, Quan X, Xia L, Fang X, Li H and Zhou B: Polymorphism in CYP24A1 is associated with lung cancer risk: A case-control study in Chinese female nonsmokers. DNA Cell Biol 38: 243-249, 2019.

9. Kato S, Naito Z, Matsuda N, Onodera H, Sakurazawa N, Yamashita N, Kanazawa Y, Fujita I, Makino H and Uchida E: Localization of cytochrome P4502E1 enzyme in normal and cancerous gastric mucosa and association with its genetic polymorphism in unoperated and remnant stomach. J Nippon Med Sch 78: 224-234, 2011.

10. Ho JC, Cheung ST, Leung KL, Ng IO and Fan ST: Decreased expression of cytochrome P450 2E1 is associated with poor prognosis of hepatocellular carcinoma. Int J Cancer 111: 494-500, 2004.

11. Gao J, Wang GJ, Wang Z, Gao N, Li J, Zhang YF, Zhou J, Zhang HX, Wen Q, Jin H and Qiao HL: High CYP2E1 activity correlates with hepatofibrogenesis induced by nitrosamines. Oncotarget 8: 112199-112210, 2017.

12. Zeng T, Zhang CL, Zhao N, Guan MJ, Xiao M, Yang R, Zhao XL, Yu LH, Zhu ZP and Xie KQ: Impairment of Akt activity by CYP2E1 mediated oxidative stress is involved in chronic ethanol-induced fatty liver. Redox Biol 14: 295-304, 2018.

13. Hernando-Rodriguez M, Rey-Barja N, Marichalar-Mendia X, Rodriguez-Tojo MJ, Acha-Sagredo A and Aguirre-Urizar JM: Role of cytochrome P-450 genetic polymorphisms in oral carcinogenesis. J Oral Pathol Med 41: 1-8, 2012.

14. Livak KJ and Schmittgen TD: Analysis of relative gene expression data using real-time quantitative PCR and the 2(-Delta Delta C(T)) method. Methods 25: 402-408, 2001.

15. Koyuncu I, Gonel A, Durgun M, Kocyigit A, Yuksekdag O and Supuran CT: Assessment of the antiproliferative and apoptotic roles of sulfonamide carbonic anhydrase IX inhibitors in HeLa cancer cell line. J Enzyme Inhib Med Chem 34: 75-86, 2019.

16. Tian X, Ji Y, Liang Y, Zhang J, Guan L and Wang C: LINC00520 targeting miR-27b-3p regulates OSMR expression level to promote acute kidney injury development through the PI3K/AKT signaling pathway. J Cell Physiol 234: 14221-14233, 2019.

17. Shen H, Zhang J, Zhang Y, Feng Q, Wang H, Li G, Jiang W and Li X: Knockdown of tripartite motif 59 (TRIM59) inhibits proliferation in cholangiocarcinoma via the PI3K/AKT/mTOR signalling pathway. Gene 698: 50-60, 2019.

18. Xing Y, Ren S, Ai L, Sun W, Zhao Z, Jiang F, Zhu Y and Piao D: ZNF692 promotes colon adenocarcinoma cell growth and metastasis by activating the PI3K/AKT pathway. Int J Oncol 54: 1691-1703, 2019.

19. Slattery ML, Mullany LE, Sakoda LC, Wolff RK, Stevens JR, Samowitz WS and Herrick JS: The PI3K/AKT signaling pathway: Associations of miRNAs with dysregulated gene expression in colorectal cancer. Mol Carcinog 57: 243-261, 2018.

20. Sivalingam K, Amirthalingam V, Ganasan K, Huang CY and Viswanadha VP: Neferine suppresses diethylnitrosamine-induced lung carcinogenesis in Wistar rats. Food Chem Toxicol 123: 385-398, 2019.

21. Potočnjak I and Domitrović R: Carvacrol attenuates acute kidney injury induced by cisplatin through suppression of ERK and PI3K/Akt activation. Food Chem Toxicol 98: 251-261, 2016.

22. Leung T, Rajendran R, Singh S, Garva R, Krstic-Demonacos M and Demonacos C: Cytochrome P450 2E1 (CYP2E1) regulates the response to oxidative stress and migration of breast cancer cells. Breast Cancer Res 15: R107, 2013.

23. Gao J, Zhou J, He XP, Zhang YF, Gao N, Tian X, Fang Y, Wen Q, JiaLJ,Jin Hand Qiao HL: Changes in cytochrome P450s-mediated drug clearance in patients with hepatocellular carcinoma in vitro and in vivo: A bottom-up approach. Oncotarget 7: 28612-28623, 2016.

24. Tonack S, Patel S, Jalali M, Nedjadi T, Jenkins RE, Goldring C, Neoptolemos J and Costello E: Tetracycline-inducible protein expression in pancreatic cancer cells: Effects of CapG overexpression. World J Gastroenterol 17: 1947-1960, 2011.

25. Na HK and Lee JY: Molecular basis of alcohol-related gastric and colon cancer. Int J Mol Sci 18: E1116, 2017.

26. Ratna A and Mandrekar P: Alcohol and cancer: Mechanisms and therapies. Biomolecules 7: E61, 2017.

27. Kamalipour S, Barzegar A, Shokrzadeh M and Nikbakhsh N: Increased Expression of CYP2E1 gene in gastric cancer may be a molecular marker for mazandaran province population. J Genet Resources 2: 130-136, 2017.

28. Tan W, Wu J, Tang H and Lin D: Expression of cytochrome P4502E1 in human liver: Relationship between genotype and phenotype in Chinese. Sci China Series C, Life Sci 44: 356-364, 2001 
29. Song L, Zhou Z, Gan Y, Li P, Xu Y, Zhang Z, Luo F, Xu J, Zhou Q and Dai F: Long noncoding RNA OIP5-AS1 causes cisplatin resistance in osteosarcoma through inducing the LPAAT $\beta / \mathrm{PI} 3 \mathrm{~K} / \mathrm{AKT} / \mathrm{mTOR}$ signaling pathway by sponging the miR-340-5p. J Cell Biochem 120: 9656-9666, 2019.

30. Vaclavikova R, Hubackova M, Stribrna-Sarmanova J, Kodet R, Mrhalova M, Novotny J, Gut I and Soucek P: RNA expression of cytochrome P450 in breast cancer patients. Anticancer Res 27: 4443-4450, 2007.

31. Schattenberg JM, Wang Y, Rigoli RM, Koop DR and Czaja MJ: CYP2E1 overexpression alters hepatocyte death from menadione and fatty acids by activation of ERK1/2 signaling. Hepatology 39: 444-455, 2004

32. Abboud-Jarrous G, Aingorn H, Ranginiguetta Z, Atzmon R, Zcharia E, Peretz T and Vlodavsky I: Heparanase processing: Site directed mutagenesis, proteolytic cleavage and activation. Cell Mol Biol 65, 2005.

33. Xie B, Wu G, Chen P, Liu K and Yuan T: Mechanism and clinical significance of HBx-mediated inhibition of CYP2E1 expression in the human HepG2 hepatoma cell line. Zhonghua Gan Zang Bing Za Zhi 22: 122-127, 2014 (In Chinese).

34. Flores-Bellver M, Bonet-Ponce L, Barcia JM, Garcia-Verdugo JM, Martinez-Gil N, Saez-Atienzar S, Sancho-Pelluz J, Jordan J, Galindo MF and Romero FJ: Autophagy and mitochondrial alterations in human retinal pigment epithelial cells induced by ethanol: Implications of 4-hydroxy-nonenal. Cell Death Dis 5: e1328, 2014.

35. García-Carracedo D, Villaronga MÁ, Álvarez-Teijeiro S, Hermida-Prado $F$ and García-Pedrero JM: Impact of $\mathrm{PI} 3 \mathrm{~K} / \mathrm{AKT} / \mathrm{mTOR}$ pathway activation on the prognosis of patients with head and neck squamous cell carcinomas. Oncotarget 7 : 29780-29793, 2016.

36. Faes S, Santoro T, Troquier L, De Souza Silva O and Dormond O Rebound pathway overactivation by cancer cells following discontinuation of PI3K or mTOR inhibition promotes cancer cell growth. Biochem Biophys Res Commun 513: 546-552, 2019.

37. Wang X, Li W, Williams M, Terada N, Alessi DR and Proud CG: Regulation of elongation factor 2 kinase by p90(RSK1) and p70 S6 kinase. EMBO J 20: 4370-4379, 2001.
38. Gang Z, Huikai L, Tao S and Nan Z: CSN5 silencing inhibits invasion and arrests cell cycle progression in human colorectal cancer SW480 and LS174T cells in vitro. Int J Clin Exp Pathol 8: 2809-2815, 2015

39. Wee KB and Aguda BD: Akt versus p53 in a network of oncogenes and tumor suppressor genes regulating cell survival and death. Biophys J 91: 857-865, 2006.

40. Yang HY, Xue LY, Xing LX, Wang J, Wang JL, Yan X and Zhang XH: Putative role of the mTOR/4E-BP1 signaling pathway in the carcinogenesis and progression of gastric cardiac adenocarcinoma. Mol Med Rep 7: 537-542, 2013.

41. Kozma SC and Thomas G: Regulation of cell size in growth, development and human disease: PI3K, PKB and S6K. Bioessays 24: 65-71, 2002.

42. Homann N: Alcohol and upper gastrointestinal tract cancer: The role of local acetaldehyde production. Addict Biol 6: 309-323, 2001.

43. Ma XM, Yoon SO, Richardson CJ, Julich K and Blenis J: SKAR links pre-mRNA splicing to mTOR/S6K1-mediated enhanced translation efficiency of spliced mRNAs. Cell 133: 303-313, 2008.

44. Gogtay NJ, Kapileshwar SR, Shah SU, Bendkhale SR, Ramakrishna S, Sridharan K, Thelma BK, Thatte UM and Kshirsagar NA: Evaluation of cytochrome P4502E1 polymorphisms in healthy adult Western Indians and patients with antituberculous drug-induced hepatotoxicity. Indian J Pharmacol 48: 42-46, 2016.

45. Takac T,Pechan T, Samajova O and Samaj J: Vesicular trafficking and stress response coupled to PI3K inhibition by LY294002 as revealed by proteomic and cell biological analysis. J Proteome Res 12: 4435-4448, 2013.

(7) $\Theta$ This work is licensed under a Creative Commons Attribution-NonCommercial-NoDerivatives 4.0 International (CC BY-NC-ND 4.0) License. 